\title{
Systemic Inflammation Response Index is a Prognostic Risk Factor in Patients with Hepatocellular Carcinoma Undergoing TACE
}

\author{
Tian-Cheng Wang $\mathbb{D}^{1,2}$ \\ Tian-Zhi $\mathrm{An}^{3}$ \\ Jun-Xiang $\mathrm{Li}^{4, *}$ \\ Peng-Fei Pang ${ }^{1} *$
}

'Department of Interventional Medicine, The Fifth Affiliated Hospital, Sun Yat-sen University, Zhuhai, Guangdong, People's Republic of China; ${ }^{2}$ Department of Radiology, The Second Xiangya Hospital of Central South University, Changsha, Hunan, People's Republic of China; ${ }^{3}$ Department of Interventional Radiology, The Affiliated Hospital of Guizhou Medical University, Guiyang, Guizhou, People's Republic of China; ${ }^{4}$ Department of Interventional Radiology, The Affiliated Cancer Hospital of Guizhou Medical University, Guiyang, Guizhou, People's Republic of China

*These authors contributed equally to this work
Correspondence: Peng-Fei Pang; Jun-Xiang Li

Email pangpf@mail.sysu.edu.cn; 357936984@qq.com
Background: Mounting evidence has shown that systemic inflammation response index (SIRI), a novel prognostic biomarker based on peripheral lymphocyte, neutrophil and monocyte counts, is associated with poor prognosis for several tumors. However, the prognostic value of SIRI in patients with hepatocellular carcinoma (HCC) undergoing transarterial chemoembolization (TACE) is elusive. Herein, we aimed to evaluate the correlation between SIRI and clinical outcomes in these patients.

Methods: A total of 194 consecutive patients who underwent TACE were included in this study. Patients were stratified into high and low SIRI groups based on the cut-off value using receiver operating characteristic (ROC) analysis. Independent risk factors for tumor response were analyzed using forward stepwise logistic regression. A one-to-one propensity score matching (PSM) was conducted to compare progression-free survival (PFS) and overall survival (OS) between low and high SIRI patients. The discriminatory power of the combination of number of tumors and SIRI in predicting initial TACE response was evaluated by ROC analysis.

Results: Patients were divided into high SIRI $(>0.88)$ and low SIRI $(\leq 0.88)$ groups. High SIRI $(p=0.003)$ and more than three tumors $(p=0.002)$ were significantly related to poorer tumor response. Moreover, the low SIRI group had longer PFS and OS than the high SIRI group (both $\mathrm{P}<0.05$ ) before and after PSM. Combination of SIRI and number of tumors can improve the predictive ability to predict initial TACE response with an area under the curve (AUC) of 0.678 .

Conclusion: Pretreatment peripheral blood SIRI was found to be an independent predictor of tumor response and clinical outcomes in patients with HCC undergoing TACE. Patients with high SIRI may have a poor prognosis.

Keywords: systemic inflammation response index, hepatocellular carcinoma, transarterial chemoembolization, biomarker, survival

\section{Introduction}

Hepatocellular carcinoma (HCC) is one of the most prevalent alimentary tumors and the third leading cause of cancer-related deaths worldwide. ${ }^{1-3}$ Potentially curative treatment for patients with HCC includes liver transplantation, liver resection and ablation. ${ }^{4-6}$ However, most patients are diagnosed at the intermediate or advanced stages of the disease and only a few of these patients can receive curative treatments. $^{7-9}$ Transarterial chemoembolization (TACE) is recommended as the standard of care for HCC patients who are ineligible for curative treatment. ${ }^{10,11}$ 
However, not all HCC patients benefit from TACE because the HCC patients selected for the TACE procedure correspond to a highly heterogeneous population, covering a wide range of tumor burdens, immune response status and liver function. ${ }^{12-14}$ Therefore, to optimize risk stratification and to predict clinical outcomes more accurately, and further inform individualized treatment methods, this population of HCC patients who undergoing TACE require effective prognostic biomarkers.

Inflammatory reaction and immune surveillance are recognized as vital hallmarks relevant to tumor progression and prognosis. ${ }^{15,16}$ Peripheral blood immune cells directly reflect the condition of the immune system. Peripheral blood leukocyte biomarkers such as the absolute counts of monocyte, neutrophil and lymphocyte, along with the neutrophil-to-lymphocyte ratio (NLR), platelet-tolymphocyte ratio (PLR) and monocyte-to-lymphocyte ratio (MLR) have been reported to be effective risk factors of prognosis in various tumor models. ${ }^{17-22}$ Low lymphocyte counts and high neutrophil or monocyte counts have been associated with poor prognosis in various cancer. Moreover, the novel systemic inflammation response index (SIRI), which integrates three types of inflammatory cells (neutrophils, monocytes, and lymphocytes), has been reported to be a potential prognostic predictor in several cancers. $^{21,23-25}$

However, the prognostic value of pretreatment SIRI in the intermediate stage or unresectable $\mathrm{HCC}$ patients receiving TACE is not well explored. Therefore, this study aimed to investigate the prognostic value of SIRI in patients undergoing TACE for HCC.

\section{Materials and Methods}

\section{Patient Selection}

This study included 857 patients with unresectable HCC who underwent TACE between January 2014 and December 2019 as an initial therapy from three medical institutions. The inclusion criteria were as follows: patients had (1) aged $>18$ years; (2) an Eastern Cooperative Oncology Group performance status of 0 ; (3) a compensated liver function (Child-Pugh class A or B) and (4) complete baseline data. The exclusion criteria were as follows: patients had (1) portal venous tumor thrombus or distant metastasis $(n=312)$; (2) lost to follow-up $(\mathrm{n}=134)$; (3) follow-up magnetic resonance (MR) or computed tomography (CT) imaging was performed after TACE beyond 3 months $(n=41)$; (4) having been treated

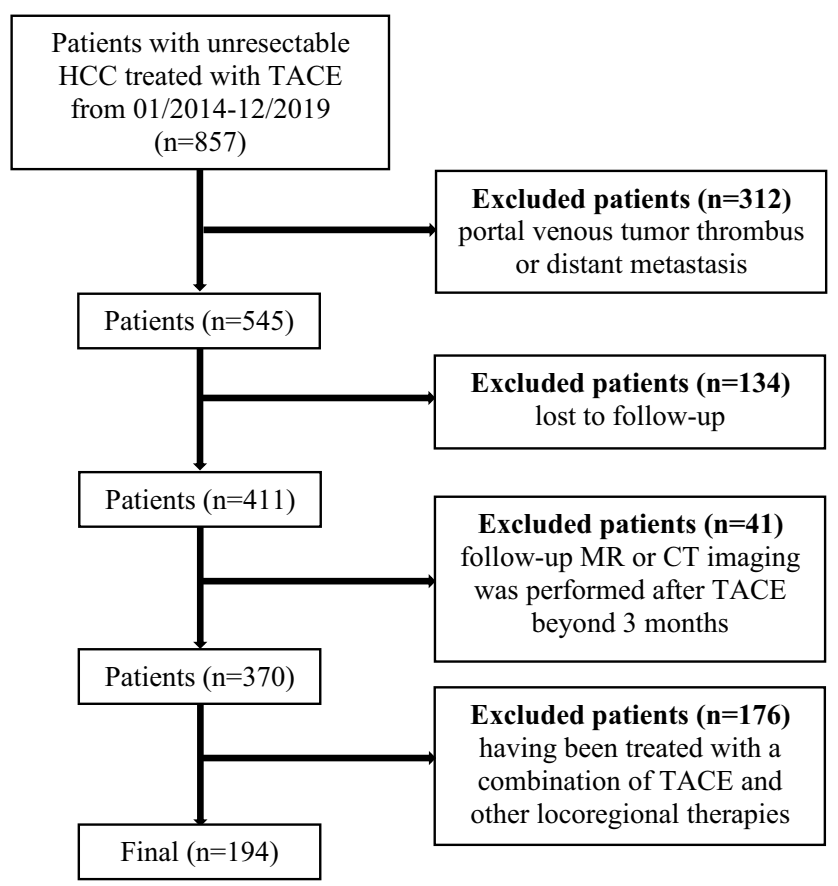

Figure I Diagram of the study population.

with a combination of TACE and other locoregional therapies such as radiofrequency or microwave ablation $(n=176)$. Finally, there were 194 patients included in the present study. The flowchart of the study population was shown in Figure 1.

This retrospective study was approved by the institutional review boards of the Second Xiangya Hospital of Central South University, the Affiliated Hospital of Guizhou Medical University and the Affiliated Cancer Hospital of Guizhou Medical University and was conducted according to the Declaration of Helsinki. The requirement for written consent was waived due to the retrospective nature of the study and the data of patients were anonymized and de-identified prior to analysis.

\section{Interventions}

The TACE procedure was considered as the initial treatment when tumors were unresectable or not feasible to radiofrequency or microwave ablation. Common hepatic artery and superior mesenteric arteriography were performed to assess tumor vascularization. A 2.7 French (Progreat, Terumo Medical Corporation) or a 2.2 French (Carnelian, Tokai Medical Products) coaxial microcatheter was used to select the tumor supply artery with the assistance of cone-beam computed tomography (CBCT). TACE procedure was performed using either drug-eluting bead (DEB) (CalliSpheres Beads, Jiangsu Hengrui Medicine 
Co., Ltd.) loaded with epirubicin or up to $20 \mathrm{~mL}$ emulsion of lipiodol (Lipiodol, Guerbet Asia Pacific Ltd.) mixed with epirubicin. The dosage of epirubicin ranged from 50 to $150 \mathrm{mg}$ in DEB-TACE and the size of DEB varied from 100 to 500 microns. The dosage of epirubicin was 50 $75 \mathrm{mg} / \mathrm{m}^{2}$ body surface area in conventional TACE. In DEB-TACE, no additional embolization was performed after the injection of 1-2 g DEB. In conventional TACE, gelfoam slurries were injected to embolize the proximal tumor-feeding artery after lipiodol-epirubicin emulsion was injected. The technical endpoint of conventional TACE or DEB-TACE procedure was defined as the decrease in subsequential arterial inflow to the tumor and tumor devascularization.

\section{Data Collection}

Patients' clinical information, including age, gender, ChildPugh class, Barcelona clinic liver cancer (BCLC) staging, underlying liver disease, initial Alpha-fetoprotein (AFP), history of resection, number of tumors, size of the largest tumor and initial embolic agents, was collected. Routine blood results within 1 week before the TACE procedure were also collected. NLR, PLR, MLR and SIRI were calculated using the following formula: NLR $=$ neutrophil count/lymphocyte count, PLR = platelet count/lymphocyte count, $\mathrm{MLR}=$ monocyte count/lymphocyte count, SIRI = neutrophil count $\times$ monocyte count/lymphocyte count.

\section{Response Evaluation and Follow-Up}

Dynamic CT/MR imaging was reviewed by two radiologists who had either 13 years or 15 years of experience in abdominal radiology. Tumor response on a patient basis was assessed based on modified response evaluation criteria in solid tumors (mRECIST) and divided into objective response (complete response and partial response) and nonobjective response (stable disease and progressive disease). The evaluation of tumor burden was based on the number of tumors, size of the largest tumor and up-to-seven criteria. Patients who were beyond up-to-seven criteria were defined as: largest tumor diameter $(\mathrm{cm})+$ number of tumors $>7 .^{26}$ Progression-free survival (PFS) was calculated from the date of diagnosis to the date of tumor progression and tumor progression was assessed based on the 2019 version of Response Evaluation Criteria in Cancer of the Liver (RECICL), which was defined as tumor enlargement of $\geq 50 \%$, excluding the area of treatment-induced necrosis in either target lesion or non-target lesion. ${ }^{27}$ Overall survival (OS) was calculated from the date of diagnosis to the date of death due to any reason or to the date of the last followup. Both PFS and OS were calculated before and after a one-to-one propensity score matching (PSM).

\section{Statistical Analysis}

The receiver operating characteristic (ROC) curve was used to determine the best cut-off value for NLR, MLR, PLR and SIRI to screen patients who responded well to TACE treatment. The corresponding value at the maximum sensitivity and specificity was defined as the optimal cut-off point. The data were shown as the mean with standard deviation (SD) or as frequencies. Independent sample $t$-test was used to compare numerical variables and Pearson's chi-squared test or Fisher's exact test was used to compare categorical variables. A forward stepwise logistic regression model was used to identify the best predictor variables using univariate and multivariate analysis. In addition, a one-to-one PSM analysis was conducted to eliminate any bias derived from confounding factors between the low and high SIRI groups. The Kaplan-Meier method and Log rank test were used to compare the OS and PFS differences between the two groups before and after PSM. Discriminatory power of the combination of SIRI and number of tumors in predicting initial TACE response was calculated by ROC curve.

Statistical analyses were performed with SPSS statistical software (SPSS version 24.0, International Business Machines Corporation) and $\mathrm{R}$ software (version 3.4.3, http://www.R-project.org). A two-tailed $P$ value of $<$ 0.05 was considered statistically significant.

\section{Result \\ Demographic, Radiological and Laboratory Characteristics}

A total of 194 patients were included (174 males and 20 females, with a mean age of $56.5 \pm 12.0$ years) in this study. All TACE procedures achieved technical success according to the Society of Interventional Radiology (SIR) guidelines. ${ }^{28}$ The diagnosis of HCC was based on pathology (biopsy, $\mathrm{n}=10$ ) or on the American Association for the Study of Liver Practice Guidelines $(n=184)$. There were 125 (64.4\%) patients with ChildPugh class A and 26 patients with Child-Pugh class B (13.4\%), 125 patients (64.4\%) in BCLC stage A and 69 patients $(35.6 \%)$ in BCLC stage B. DEB-TACE was initially performed in 29 patients $(29 / 194,14.9 \%)$, and 
Table I The Demographic, Radiological and Laboratorial Characteristics of the Patients

\begin{tabular}{|c|c|c|c|c|}
\hline Characteristics & Total $(n=194)$ & Low SIRI $(n=86)$ & High SIRI $(n=108)$ & $P$ value \\
\hline Age (years) & $56.5 \pm 12.0$ & $55.6 \pm 11.2$ & $57.1 \pm 12.6$ & 0.383 \\
\hline Gender (\%) & & & & 0.049 \\
\hline Male & 174 (89.7\%) & 73 (84.9\%) & 101 (93.5\%) & \\
\hline Female & $20(10.3 \%)$ & $13(15.1 \%)$ & $7(6.5 \%)$ & \\
\hline Child-Pugh class (\%) & & & & 0.294 \\
\hline A & 168 (86.6\%) & $72(83.7 \%)$ & $96(88.9 \%)$ & \\
\hline B & $26(13.4 \%)$ & $14(16.3 \%)$ & 12 (II.1\%) & \\
\hline BCLC stage (\%) & & & & 0.435 \\
\hline A & $125(64.4 \%)$ & $58(67.4 \%)$ & $67(62.0 \%)$ & \\
\hline B & 69 (35.6\%) & $28(32.6 \%)$ & $4 \mathrm{I}(38.0 \%)$ & \\
\hline Underlying liver disease (\%) & & & & 0.279 \\
\hline HBV & $152(78.4 \%)$ & 66 (76.8\%) & $86(79.6 \%)$ & \\
\hline Other & $16(8.2 \%)$ & $10(11.6 \%)$ & $6(5.6 \%)$ & \\
\hline None & $26(13.4 \%)$ & $10(11.6 \%)$ & $16(14.8 \%)$ & \\
\hline Initial AFP (\%) & & & & 0.153 \\
\hline$\leq 400 \mathrm{ug} / \mathrm{L}$ & $74(38.1 \%)$ & $28(32.6 \%)$ & $46(42.6 \%)$ & \\
\hline$>400$ ug/L & $120(61.9 \%)$ & $58(67.4 \%)$ & $62(57.4 \%)$ & \\
\hline History of resection (\%) & & & & 0.850 \\
\hline Presence & 15 (7.7\%) & $7(8.1 \%)$ & $8(7.4 \%)$ & \\
\hline Absence & 179 (92.3\%) & 79 (91.9\%) & $100(92.6 \%)$ & \\
\hline Number of tumors (\%) & & & & 0.655 \\
\hline Solitary & $120(61.9 \%)$ & $55(64.0 \%)$ & $65(60.2 \%)$ & \\
\hline $2-3$ & 44 (22.7\%) & $20(23.3 \%)$ & $24(22.2 \%)$ & \\
\hline$>3$ & $30(15.4 \%)$ & $11(12.7 \%)$ & $19(17.6 \%)$ & \\
\hline Size of the largest tumor (\%) & & & & $<0.001$ \\
\hline$\leq 50 \mathrm{~mm}$ & 69 (35.6\%) & 48 (55.8\%) & 21 (19.4\%) & \\
\hline$>50 \mathrm{~mm}$ & $125(64.4 \%)$ & 38 (44.2\%) & 87 (80.6\%) & \\
\hline Up-to-seven criteria (\%) & & & & $<0.001$ \\
\hline Within & 71 (36.6\%) & 49 (57.0\%) & $22(20.4 \%)$ & \\
\hline Beyond & $123(63.4 \%)$ & 37 (43.0\%) & $86(79.6 \%)$ & \\
\hline Initial embolic agents (\%) & & & & 0.729 \\
\hline Lipiodol & $165(85.1 \%)$ & 74 (86.0\%) & 91 (84.3\%) & \\
\hline DEB & $29(14.9 \%)$ & $12(14.0 \%)$ & $17(15.7 \%)$ & \\
\hline Platelet $\left(\times 10^{9} / \mathrm{L}\right)$ & $151.8 \pm 84.8$ & $109.8 \pm 65.1$ & $185.2 \pm 84.1$ & $<0.001$ \\
\hline Neutrophils $\left(\times 10^{9} / \mathrm{L}\right)$ & $3.6 \pm 2.2$ & $2.2 \pm 0.9$ & $4.6 \pm 2.4$ & $<0.001$ \\
\hline Monocytes $\left(\times 10^{9} / \mathrm{L}\right)$ & $0.5 \pm 0.3$ & $0.3 \pm 0.1$ & $0.6 \pm 0.3$ & $<0.001$ \\
\hline Lymphocytes $\left(\times 10^{9} / \mathrm{L}\right)$ & $1.4 \pm 0.8$ & $1.3 \pm 0.7$ & $1.4 \pm 0.8$ & 0.774 \\
\hline Initial TACE response (\%) & & & & 0.002 \\
\hline Objective response & $126(64.9 \%)$ & 66 (76.7\%) & $60(55.5 \%)$ & \\
\hline Non-objective response & $68(35.1 \%)$ & $20(23.3 \%)$ & 48 (44.5\%) & \\
\hline
\end{tabular}

Abbreviations: SIRI, systemic inflammation response index; HBV, hepatitis B virus; AFP, alpha-fetoprotein; BCLC, Barcelona Clinic Liver Cancer; DEB, drug-eluting beads.

conventional chemoembolization was initially performed in 165 patients $(165 / 194,85.1 \%)$. There were 120 patients $(61.9 \%)$ with initial AFP $>400 \mathrm{ug} / \mathrm{L}$ and 74 patients $(38.1 \%)$ with AFP $\leq 400 \mathrm{ug} / \mathrm{L}$. The sizes of the largest tumors were $\leq 50 \mathrm{~mm}$ in 69 patients $(35.6 \%)$ and $>50 \mathrm{~mm}$ in 125 patients $(64.4 \%)$. Seventy-one patients 


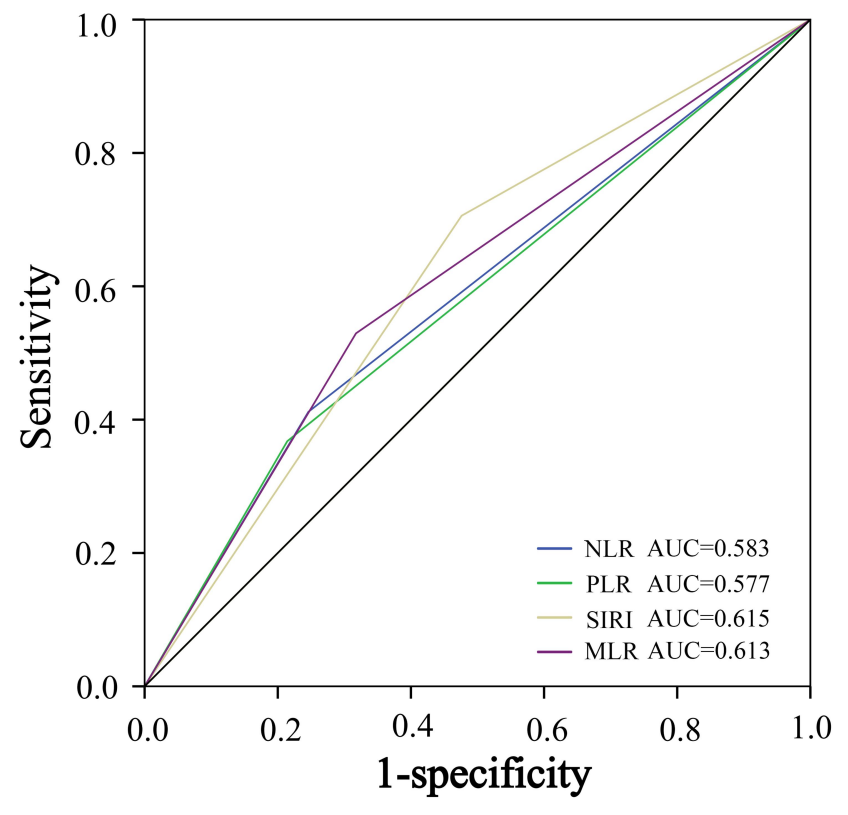

Figure 2 Comparison of the AUCs for NLR, MLR, PLR and SIRI in predicting tumor response in HCC patients undergoing TACE.

Abbreviations: AUCs, area under curves; NLR, neutrophil-to-lymphocyte ratio; MLR, monocyte-to-lymphocyte; PLR, platelet-to-lymphocyte ratio; SIRI, systemic inflammation response index; HCC, hepatocellular carcinoma; TACE, transarterial chemoembolization.

$(36.6 \%)$ were within up-to-seven criteria and 125 patients $(64.4 \%)$ were beyond the up-to-seven criteria. Detailed demographic, radiological and laboratory characteristics are summarized in Table 1.

\section{Potential Predictive Factors of Initial TACE Response}

One hundred twenty-six $(64.9 \%)$ patients had objective response and 68 (35.1\%) patients had nonobjective response. The ROC curve was performed to determine the best biomarker and cutoff points of different biomarkers. The optimal cut-off points of NLR, MLR, PLR and SIRI were $3.2,0.35,162$ and 0.88 , respectively. The area under the curves (AUCs) of NLR, MLR, PLR and SIRI were 0.583 (positive predictive value (PPV): $47.5 \%$, negative predictive value (NPV): 70.4\%), 0.606 (PPV: 47.4\%, NPV: 72.9\%), 0.577 (PPV: 48.1\%, NPV: 69.7\%) and 0.615 (PPV: 44.4\%, NPV: 76.6\%), respectively (Figure 2). In univariate analysis, the initial TACE objective response was associated with age $(\mathrm{p}=0.025)$, BCLC stage $(\mathrm{p}=$ $0.014), \operatorname{NLR} \leq 3.2(\mathrm{p}=0.017), \operatorname{MLR} \leq 0.035(\mathrm{p}=0.035)$, PLR $\leq 162(p=0.021)$, SIRI $\leq 0.88(p=0.021)$, solitary tumor $(\mathrm{p}=0.002)$, tumor size $\leq 50 \mathrm{~mm}(\mathrm{p}=0.010)$ and within up-to-seven criteria $(p=0.006)$. A forward stepwise multivariate analysis was performed using significant risk factors determined in the univariate analysis where solitary tumor $(\mathrm{p}=0.002$; odds ratio $=1.920,95 \%$ CI 1.279 $2.882)$ and $\operatorname{SIRI} \leq 0.88(\mathrm{p}=0.003$; odds ratio $=3.251$, 95\% CI 1.536-6.883) were independent predictors associated with objective response (Table 2).

\section{Comparison of Survival Analysis Between Low SIRI and High SIRI Patients}

According to the results obtained using the logistics regression model, NLR, MLR, PLR and SIRI correlated with TACE response in the univariate analysis, while only SIRI significantly correlated with TACE response in multivariate analysis. Therefore, the Log rank test was performed to determine the difference in PFS and OS between patients with low and high SIRI. Patients with low SIRI were 86 (46.2\%) and those with high SIRI were 108 (53.8\%). Compared to the low SIRI cohort, high SIRI patients were often males ( $93.5 \%$ vs $84.9 \%$ ), had a tumor size of $>50 \mathrm{~mm}$ at diagnosis ( $80.6 \%$ vs $44.2 \%)$ and beyond up-to-seven criteria (79.6\% vs $43.0 \%$ ) (Table 1). Kaplan-Meier survival curves of PFS and OS in patients with low SIRI and high SIRI are shown in Figure 2. Compared with high SIRI group, the low SIRI group had a significantly longer median PFS (low SIRI, 392.0 days [95\% CI 237.4-547.6] vs high SIRI, 216.0 days $[95 \%$ CI 159.6-272.4]; $\mathrm{p}=0.018$ ) (Figure 3A) and OS (low SIRI, 987.0 days [95\% CI 589.6-1384.4] vs high SIRI, 500.0 days [95\% CI $400.8-$ 599.2]; $\mathrm{p}=0.001$ ) (Figure 3B). Moreover, to reduce selection bias from confounding factors between patients with low SIRI and those with high SIRI, a one-to-one PSM analysis was performed. After PSM, 124 patients were enrolled, of which 62 had low SIRI and 62 had high SIRI. There was no difference in baseline characteristics between the two groups after PSM (Table 3). Similarly, compared with high SIRI group, the low SIRI group had significantly longer median PFS (low SIRI, 467.0 days [95\% CI 266.5667.5] vs high SIRI, 247.0 days [95\% CI 117.0-377.0]; $\mathrm{p}=$ 0.032) (Figure 4A) and OS (low SIRI, 1046.0 days [95\% CI 604.0-1538.0] vs high SIRI, 425.0 days [95\% CI 304.5545.5]; $\mathrm{p}<0.001$ ) (Figure 4B).

\section{Discriminatory Power of the Combination of SIRI and Number of Tumors in Predicting Initial TACE Response}

The ROC curves of the SIRI, number of tumors and the combination of SIRI and number of tumors to predict 
Table 2 Assessment of Potential Risk Factors of or to Initial TACE

\begin{tabular}{|c|c|c|c|c|}
\hline \multirow[t]{2}{*}{ Characteristics } & \multirow{2}{*}{$\begin{array}{l}\text { OR to Initial TACE } \\
(n=126)\end{array}$} & \multirow{2}{*}{$\begin{array}{l}\text { Non-OR to Initial TACE } \\
(n=68)\end{array}$} & \multicolumn{2}{|l|}{$P$ value } \\
\hline & & & Univariate & Multivariate \\
\hline Age (years) & $57.9 \pm 11.8$ & $53.9 \pm 11.8$ & 0.025 & - \\
\hline $\begin{array}{l}\text { Gender }(\%) \\
\text { Male } \\
\text { Female }\end{array}$ & $\begin{array}{l}112(88.9 \%) \\
14(11.1 \%)\end{array}$ & $\begin{array}{l}62(91.2 \%) \\
6(8.8 \%)\end{array}$ & 0.617 & - \\
\hline $\begin{array}{l}\text { Child Pugh class (\%) } \\
\text { A } \\
\text { B }\end{array}$ & $\begin{array}{l}107(84.9 \%) \\
19(15.1 \%)\end{array}$ & $\begin{array}{l}61(89.7 \%) \\
7(10.3 \%)\end{array}$ & 0.351 & - \\
\hline $\begin{array}{l}\text { BCLC stage (\%) } \\
\text { A } \\
\text { B }\end{array}$ & $\begin{array}{l}89(70.6 \%) \\
37(29.4 \%)\end{array}$ & $\begin{array}{l}36(52.9 \%) \\
32(47.1 \%)\end{array}$ & 0.014 & - \\
\hline $\begin{array}{l}\text { NLR } \\
\quad \leq 3.2 \\
>3.2\end{array}$ & $\begin{array}{l}95(75.4 \%) \\
31(24.6 \%)\end{array}$ & $\begin{array}{l}40(58.8 \%) \\
28(41.2 \%)\end{array}$ & 0.017 & - \\
\hline $\begin{array}{l}\text { MLR } \\
\quad \leq 0.35 \\
>0.35\end{array}$ & $\begin{array}{l}86(68.3 \%) \\
40(31.7 \%)\end{array}$ & $\begin{array}{l}32(47.1 \%) \\
36(52.9 \%)\end{array}$ & 0.035 & - \\
\hline $\begin{array}{l}\text { PLR } \\
\quad \leq 162 \\
>162\end{array}$ & $\begin{array}{l}99(78.6 \%) \\
27(21.4 \%)\end{array}$ & $\begin{array}{l}43(63.2 \%) \\
25(36.8 \%)\end{array}$ & 0.021 & - \\
\hline $\begin{array}{l}\text { SIRI } \\
\quad \leq 0.88 \\
>0.88\end{array}$ & $\begin{array}{l}66(52.4 \%) \\
60(47.6 \%)\end{array}$ & $\begin{array}{l}20(29.4 \%) \\
48(70.6 \%)\end{array}$ & 0.002 & $\begin{array}{l}0.003 \text { (OR, 2.623; 95\% Cl: I.376- } \\
5.002)\end{array}$ \\
\hline $\begin{array}{l}\text { Underlying liver disease } \\
\text { (\%) } \\
\text { HBV } \\
\text { Other } \\
\text { None }\end{array}$ & $\begin{array}{l}99(78.6 \%) \\
9(7.1 \%) \\
18(14.3 \%)\end{array}$ & $\begin{array}{l}53(77.9 \%) \\
7(10.3 \%) \\
8(11.8 \%)\end{array}$ & 0.690 & - \\
\hline $\begin{array}{l}\text { Initial AFP (\%) } \\
\quad \leq 400 \text { ug/L } \\
>400 \text { ug/L }\end{array}$ & $\begin{array}{l}45(35.7 \%) \\
81(64.3 \%)\end{array}$ & $\begin{array}{l}29(42.6 \%) \\
39(57.4 \%)\end{array}$ & 0.343 & - \\
\hline $\begin{array}{l}\text { History of resection (\%) } \\
\text { Presence } \\
\text { Absence }\end{array}$ & $\begin{array}{l}I 1(8.7 \%) \\
115(91.3 \%)\end{array}$ & $\begin{array}{l}4(5.9 \%) \\
64(94.1 \%)\end{array}$ & 0.479 & - \\
\hline $\begin{array}{l}\text { Number of tumors (\%) } \\
\text { Solitary } \\
2-3 \\
>3\end{array}$ & $\begin{array}{l}86(68.3 \%) \\
29(23.0 \%) \\
I I(8.7 \%)\end{array}$ & $\begin{array}{l}34(50.0 \%) \\
15(22.1 \%) \\
19(27.9 \%)\end{array}$ & 0.002 & $\begin{array}{l}0.002 \text { (OR, I.920; } 95 \% \mathrm{Cl}: 1.279- \\
2.882)\end{array}$ \\
\hline $\begin{array}{l}\text { Size of the largest tumor } \\
\begin{array}{l}\text { (\%) } \\
\leq 50 \mathrm{~mm} \\
>50 \mathrm{~mm}\end{array}\end{array}$ & $\begin{array}{l}53(42.1 \%) \\
73(57.9 \%)\end{array}$ & $\begin{array}{l}16(23.5 \%) \\
52(76.5 \%)\end{array}$ & 0.01 & - \\
\hline
\end{tabular}

(Continued) 
Table 2 (Continued).

\begin{tabular}{|l|l|l|l|l|}
\hline Characteristics & $\begin{array}{l}\text { OR to Initial TACE } \\
(\mathbf{n}=126)\end{array}$ & $\begin{array}{l}\text { Non-OR to Initial TACE } \\
(\mathbf{n = 6 8 )}\end{array}$ & \multicolumn{2}{l|}{ P value } \\
\cline { 3 - 5 } & & & Univariate & Multivariate \\
\hline $\begin{array}{l}\text { Up-to-seven criteria (\%) } \\
\text { Within } \\
\text { Beyond }\end{array}$ & $\begin{array}{l}55(43.7 \%) \\
71(56.3 \%)\end{array}$ & $\begin{array}{l}16(23.5 \%) \\
52(76.5 \%)\end{array}$ & 0.006 & - \\
\hline $\begin{array}{l}\text { Initial embolic agent (\%) } \\
\text { Lipiodol }\end{array}$ & $107(84.9 \%)$ & $58(85.3 \%)$ & 0.945 & - \\
DEB & $19(15.1 \%)$ & $10(14.7 \%)$ & & \\
\hline
\end{tabular}

Abbreviations: OR, objective response; TACE, transarterial chemoembolization; NLR, neutrophil to lymphocyte ratio; MLR, monocyte-to-lymphocyte ratio; PLR, platelet to lymphocyte ratio; SIRI, systemic inflammation response index; HBV, hepatitis B virus; BCLC, Barcelona Clinic Liver Cancer; AFP, alpha-fetoprotein; DEB, drug-eluting beads.

initial TACE response were shown in Figure 5. The corresponding AUCs were 0.615 (PPV: 44.4\%, NPV: 76.6\%), 0.614 (PPV: 63.3\%, NPV: 70.1\%) and 0.678 (PPV: 73.4\%, NPV: 69.1\%), respectively (Figure 5). Compared to the discriminatory power of SIRI or number of tumors, combination of SIRI and number of tumors showed an outperformed discriminatory power in predicting initial TACE response.

\section{Discussion}

TACE is the standard therapy for patients with an intermediate stage or unresectable HCC. ${ }^{10,11}$ However, due to the heterogeneity of HCC, not all HCC patients benefit from TACE and the OS of patients treated by TACE ranges from 12 months to 47 months. ${ }^{9,29,30}$ Therefore, identifying effective prognostic factors for HCC patients undergoing TACE is urgent for accurate prediction of clinical outcomes. In the present study, inflammatory biomarkers NLR, MLR, PLR, and SIRI were analyzed by the ROC curve. The cut-off point of SIRI was 0.88 and the AUC of SIRI was superior to that of NLR, MLR and PLR in the entire study population. Univariate and multivariate analysis revealed that high SIRI was negatively correlated with tumor response and positively correlated with tumor burden (tumor size $>50 \mathrm{~mm}$ ) and beyond up-to-seven criteria. In addition, high SIRI remained an unfavorable prognostic factor for OS and PFS in Kaplan-Meier survival analysis before and after PSM. Although the cut-off values of SIRI vary among different types of cancers, our results were consistent with those of previous studies that reported a high pretreatment level of SIRI to be an independent unfavorable prognostic factor for malignancies. ${ }^{16,25,31}$
A
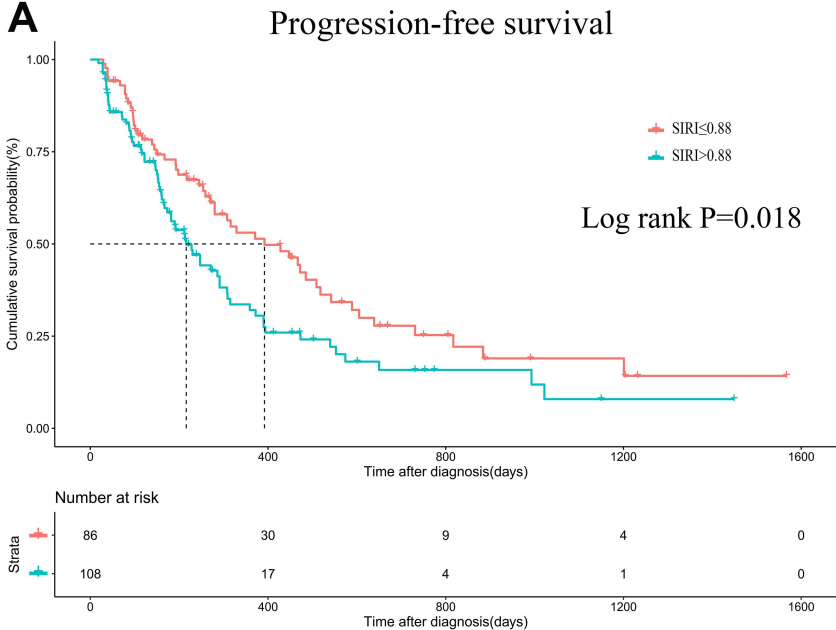

B Overall survival

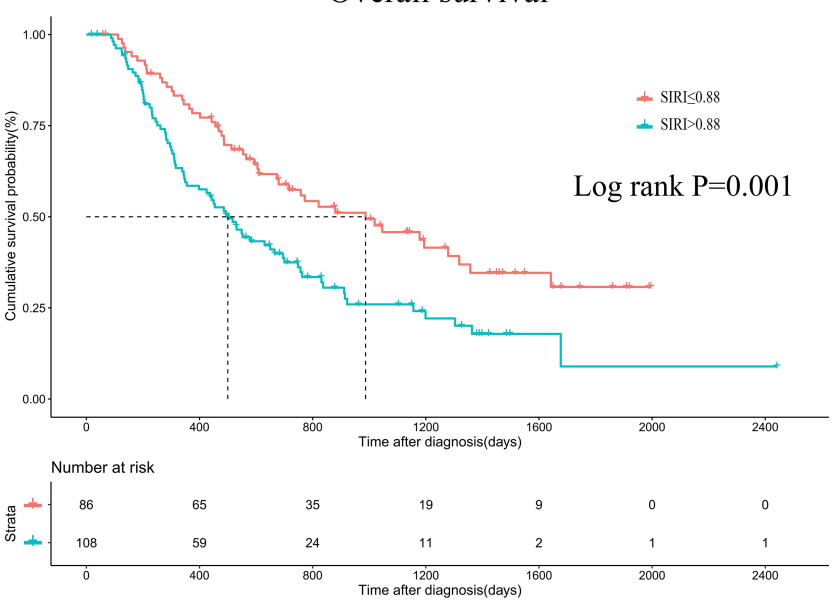

Figure 3 Log rank tests were used to compare PFS (A) and OS (B) between high SIRI and low SIRI groups before PSM.

Abbreviations: PFS, progression-free survival; OS, overall survival; SIRI, systemic inflammation response index; PSM, propensity score matching. 
Table 3 Demographic, Radiological and Laboratorial Characteristics of the Patients After Propensity Score Matching

\begin{tabular}{|c|c|c|c|}
\hline Characteristics & Low SIRI $(n=62)$ & High SIRI (n=62) & $P$ value \\
\hline Age (years) & $56.5 \pm 10.4$ & $59.5 \pm 10.7$ & 0.118 \\
\hline Gender (\%) & & & 0.769 \\
\hline Male & 55 (84.9\%) & $56(93.5 \%)$ & \\
\hline Female & $7(15.1 \%)$ & $6(6.5 \%)$ & \\
\hline Child-Pugh class (\%) & & & 0.308 \\
\hline A & 51 (82.3\%) & 55 (88.7\%) & \\
\hline B & II (I7.7\%) & 7 (11.3\%) & \\
\hline BCLC stage (\%) & & & 0.448 \\
\hline A & 39 (62.9\%) & $43(69.4 \%)$ & \\
\hline B & $23(37.1 \%)$ & $19(30.6 \%)$ & \\
\hline Underlying liver disease (\%) & & & 0.515 \\
\hline $\mathrm{HBV}$ & 48 (77.4\%) & 46 (74.2\%) & \\
\hline Other & $8(12.9 \%)$ & $6(9.7 \%)$ & \\
\hline None & $6(9.7 \%)$ & $10(16.1 \%)$ & \\
\hline Initial AFP (\%) & & & 0.137 \\
\hline$\leq 400$ ug/L & 19 (30.6\%) & 27 (43.5\%) & \\
\hline$>400$ ug/L & 43 (69.4\%) & $35(56.5 \%)$ & \\
\hline History of resection (\%) & & & 0.299 \\
\hline Presence & $6(9.7 \%)$ & $3(4.8 \%)$ & \\
\hline Absence & 56 (90.3\%) & 59 (95.2\%) & \\
\hline Number of tumors (\%) & & & 0.408 \\
\hline Solitary & $36(58.1 \%)$ & 41 (66.1\%) & \\
\hline $2-3$ & 15 (24.2\%) & I5 (24.2\%) & \\
\hline$>3$ & II (I7.7\%) & $6(9.7 \%)$ & \\
\hline Size of the largest tumor (\%) & & & 0.575 \\
\hline$\leq 50 \mathrm{~mm}$ & $24(38.7 \%)$ & 21 (33.9\%) & \\
\hline$>50 \mathrm{~mm}$ & $38(61.3 \%)$ & $41(66.1 \%)$ & \\
\hline Up-to-seven criteria (\%) & & & 0.579 \\
\hline Within & $25(40.3 \%)$ & $22(35.5 \%)$ & \\
\hline Beyond & 37 (59.7\%) & $40(64.5 \%)$ & \\
\hline Initial embolic agents (\%) & & & 0.625 \\
\hline Lipiodol & 51 (82.3\%) & $53(85.5 \%)$ & \\
\hline DEB & II (I7.7\%) & $9(14.5 \%)$ & \\
\hline Initial TACE response (\%) & & & 0.036 \\
\hline Objective response & 47 (75.8\%) & $36(58.1 \%)$ & \\
\hline Non-objective response & $15(24.2 \%)$ & 26 (4I.9\%) & \\
\hline
\end{tabular}

Abbreviations: SIRI, systemic inflammation response index; HBV, hepatitis B virus; BCLC, Barcelona Clinic Liver Cancer; AFP, alpha-fetoprotein; DEB, drug-eluting beads.

Inflammation is a critical hallmark of cancer. Over the past decade, the complicated nature of tumor cells and their reciprocal interactions with the associated microenvironment have been increasingly known to be of significance in the regulation of tumorigenesis and tumor progression. ${ }^{15}$ Because the majority of HCC develops in chronically inflamed cirrhotic livers caused by hepatitis virus or alcohol, HCC is particularly remarkable 

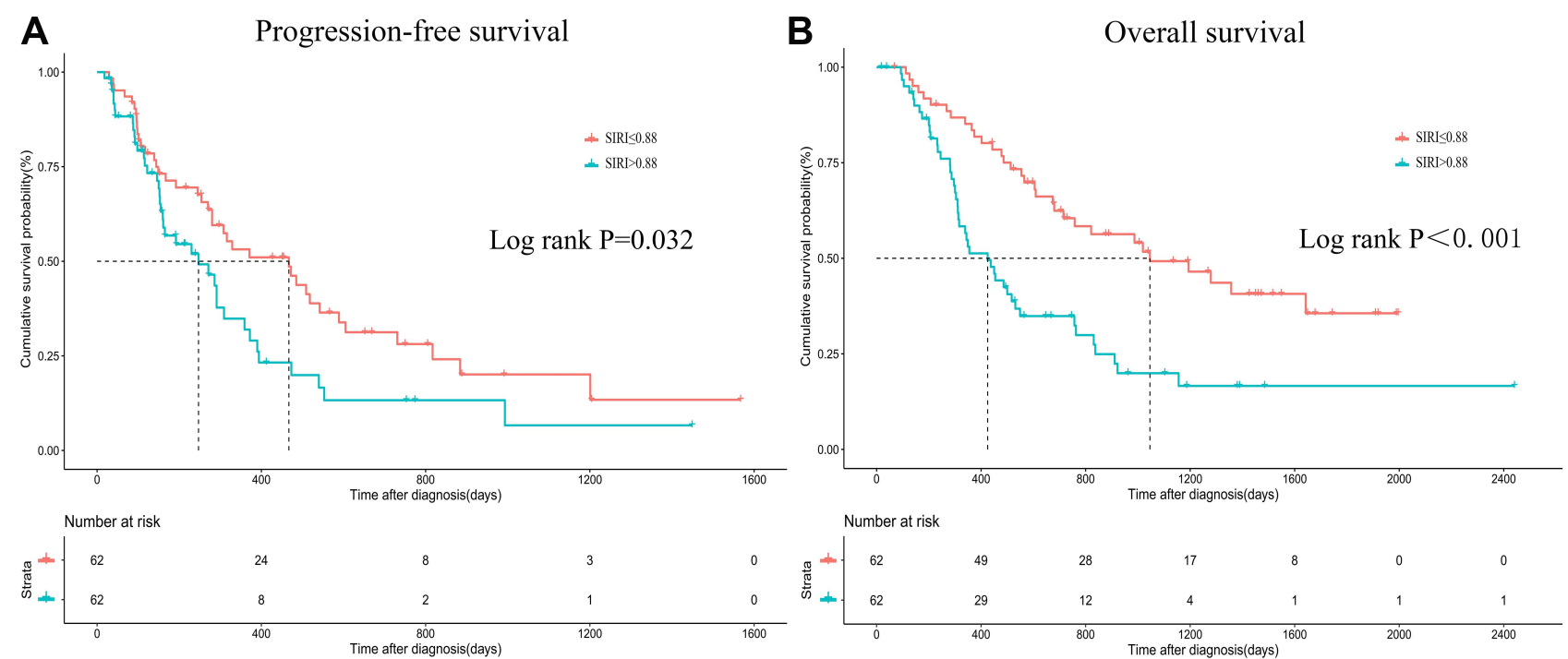

Figure 4 Log rank tests were used to compare PFS (A) and OS (B) between high SIRI and low SIRI groups after PSM.

Abbreviations: PFS, progression-free survival; OS, overall survival; SIRI, systemic inflammation response index; PSM, propensity score matching.

concerning the interaction of tumors with the immune system. $^{32,33}$ Moreover, chronic hepatopathy is hypothesized to generate a pro-inflammatory environment, which enables tumor progression and potentially participates in the resistance of tumor cells to chemotherapy and immunotherapy. ${ }^{34-36}$ Therefore, inflammation has been

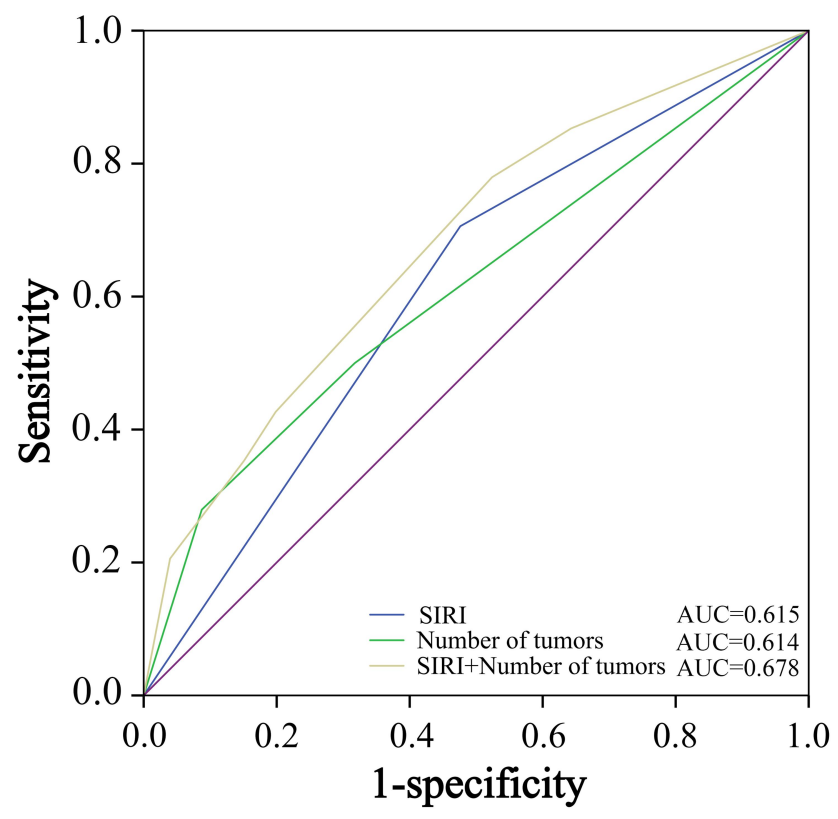

Figure 5 Comparison of the AUCs for SIRI, number of tumors and the combination of SIRI and number of tumors in predicting tumor response in $\mathrm{HCC}$ patients undergoing TACE.

Abbreviations: AUCs, area under curves; SIRI, systemic inflammation response index; HCC, hepatocellular carcinoma; TACE, transarterial chemoembolization. introduced as a critical hallmark of $\mathrm{HCC}$ and it promotes immunosuppression, tumor proliferation, tumor invasion and tumor metastasis.

Inflammation associated with cancer progression is triggered by various blood immune cells, including neutrophils, monocytes and T/B lymphocytes. ${ }^{15}$ Neutrophils have been proved to have cancer-promoting properties. They can migrate to the tumor microenvironment and secrete reactive oxygen species, which can trigger genetic instability and lead to cellular DNA damage, causing carcinogenesis and promoting tumor progression. ${ }^{37}$ Peripheral blood monocytes can be recruited to the tumor stroma and differentiate into tumorassociated macrophages. These macrophages can promote tumor progression and therapeutic resistance through the following pathways. First, overexpression of CXCL6, CCL2, CCL17, CCL24 and recruiting these chemokines, myeloid-derived inhibitory cells and regulatory $\mathrm{T}$ cells to cause immunosuppression. ${ }^{38}$ Second, strengthening the function of GM-CSF, IL-6 and other cancer-promoting cytokines. ${ }^{39}$ Third, upregulating the expression of PD-L1, CTLA4 and other common inhibitors to cause immunosuppression. ${ }^{40}$ Finally, secreting matrix metalloproteinases such as MMP-2 or MMP-9 and enhancing the ability of tumor invasion and metastasis by regulating angiogenesis and epithelial proliferation. Peripheral blood lymphocytes can be recruited to the tumor microenvironment and infiltration by lymphocytes can promote cytotoxic cell death and inhibit tumor cell proliferation and migration. ${ }^{41}$ For example, $\mathrm{CD} 8^{+}$cytotoxic lymphocytes can recognize tumor antigens 
which may be exposed thoroughly after TACE and directly kill tumor cells by producing cytotoxins such as perforin and granzyme or inducing tumor cell apoptosis through the FasFasL pathway. $^{42}$

Previous studies have shown pretreatment neutrophil, lymphocyte, monocyte counts, NLR and MLR were associated with prognosis in HCC patients. ${ }^{18,36,43}$ Zhu et al reported that MLR could predict sorafenib response, and a higher MLR was correlated with a worse prognosis in advanced HCC patients. ${ }^{43}$ Hong et al showed that pretreatment peripheral neutrophil, lymphocyte and monocyte counts were independently associated with HCC patients' outcomes. ${ }^{18}$ Schobert et al demonstrated that high baseline NLR was predictor of poorer tumor response and shorter PFS in HCC patients undergoing DEB-TACE. ${ }^{36}$ However, all the variables above contain only one or two cell types and SIRI, which is composed of three types of cells including monocytes, neutrophils and lymphocytes, may display a better predictive ability in HCC patients. To date, SIRI has already shown its predictive value in several cancers. Qi et al reported that SIRI can be used to predict the prognosis of patients with pancreatic adenocarcinomas who receive chemotherapy and patients with $\mathrm{SIRI} \geq 1.8$ had a shorter time to disease progression and OS. ${ }^{16}$ Feng et al showed that SIRI was an independent predictor of PFS and OS in locally advanced nasopharyngeal carcinoma patients, and patients with SIRI $\geq 0.86$ had a worse prognosis. ${ }^{21}$ These findings were consistent with our results and we found that HCC patients undergoing TACE with SIRI $>0.88$ had a lower objective response rate and a worse prognosis. The clinical significance of our study is that it provides a relatively convenient and noninvasive biomarker for predicting objective response rate and long-term survival of HCC patients treated by TACE. Furthermore, SIRI can be used in combination with other clinical predictors to improve the predictive efficiency.

However, the present study has some inherent limitations. First, our sample size was relatively small and thus may be subject to selection and statistical bias. Second, inflammatory biomarkers NLR, PLR, MLR and SIRI could be influenced by various factors, such as acute infection, which may have biased the results. Third, SIRI was only calculated before TACE in our study. However, the dynamic change of SIRI between pre- and post-TACE may provide more information about the host's inflammatory and immune response status. Therefore, further studies should be conducted to validate this hypothesis.

\section{Conclusion}

In conclusion, the present study demonstrates that high pretreatment SIRI level is an independent risk factor for lower tumor response rate and worse prognosis in HCC patients undergoing TACE. This biomarker or the combination of this biomarker with other clinical predictors can easily be implemented in clinical practice and may provide a novel paradigm for personalized application of TACE therapy.

\section{Data Sharing Statement}

The datasets used and analyzed during the present study are available from the corresponding author (Jun-Xiang Li) on reasonable request.

\section{Funding}

There is no funding to report.

\section{Disclosure}

The authors report no conflicts of interest in this work.

\section{References}

1. Arizumi T, Ueshima K, Chishina H, et al. Validation of the criteria of transcatheter arterial chemoembolization failure or refractoriness in patients with advanced hepatocellular carcinoma proposed by the LCSGJ. Oncology. 2014;87(Suppl 1):32-36. doi:10.1159/000368143

2. Young S, Craig P, Golzarian J. Current trends in the treatment of hepatocellular carcinoma with transarterial embolization: a cross-sectional survey of techniques. Eur Radiol. 2019;29 (6):3287-3295. doi:10.1007/s00330-018-5782-7

3. Witjes CD, Polak WG, Verhoef C, et al. Increased alpha-fetoprotein serum level is predictive for survival and recurrence of hepatocellular carcinoma in non-cirrhotic livers. Dig Surg. 2012;29(6):522-528. doi: $10.1159 / 000348669$

4. Arizumi T, Ueshima K, Minami T, et al. Effectiveness of sorafenib in patients with transcatheter arterial chemoembolization (TACE) refractory and intermediate-stage hepatocellular carcinoma. Liver Cancer. 2015;4(4):253-262. doi:10.1159/000367743

5. Belghiti J, Fuks D. Liver resection and transplantation in hepatocellular carcinoma. Liver Cancer. 2012;1(2):71-82. doi:10.1159/ 000342403

6. Lin SM. Local ablation for hepatocellular carcinoma in Taiwan. Liver Cancer. 2013;2(2):73-83. doi:10.1159/000343843

7. Nishikawa H, Kita R, Kimura T, Osaki Y. Transcatheter arterial embolic therapies for hepatocellular carcinoma: a literature review. Anticancer Res. 2014;34(12):6877-6886.

8. Peng Z, Chen S, Xiao H, et al. Microvascular invasion as a predictor of response to treatment with sorafenib and transarterial chemoembolization for recurrent intermediate-stage hepatocellular carcinoma. Radiology. 2019;292(1):237-247. doi:10.1148/radiol.2019181818

9. Luedemann WM, Geisel D, Gebauer B, et al. Comparing HCC arterial tumour vascularisation on baseline imaging and after lipiodol cTACE: how do estimations of enhancing tumour volumes differ on contrast-enhanced MR and CT? Eur Radiol. 2020;30(3):1601-1608. doi:10.1007/s00330-019-06430-2 
10. Khalaf MH, Sundaram V, AbdelRazek MM, et al. A predictive model for postembolization syndrome after transarterial hepatic chemoembolization of hepatocellular carcinoma. Radiology. 2019;290 (1):254-261. doi:10.1148/radiol.2018180257

11. Guo JG, Zhao LP, Rao YF, et al. Novel multimodal analgesia regimen improves post-TACE pain in patients with hepatocellular carcinoma. Hepatobiliary Pancreat Dis Int. 2018;17(6):510-516. doi:10.1016/j. hbpd.2018.08.001

12. Kudo M, Han KH, Ye SL, et al. A changing paradigm for the treatment of intermediate-stage hepatocellular carcinoma: Asia-pacific primary liver cancer expert consensus statements. Liver Cancer. 2020;9(3):245-260. doi:10.1159/000507370

13. Bruix J, Reig M, Sherman M. Evidence-based diagnosis, staging, and treatment of patients with hepatocellular carcinoma. Gastroenterology. 2016;150(4):835-853. doi:10.1053/j. gastro.2015.12.041

14. Kudo M, Matsui O, Izumi N, et al. JSH consensus-based clinical practice guidelines for the management of hepatocellular carcinoma: 2014 update by the liver cancer study group of Japan. Liver Cancer. 2014;3(3-4):458-468. doi:10.1159/000343875

15. Mantovani A, Allavena P, Sica A, Balkwill F. Cancer-related inflammation. Nature. 2008;454(7203):436-444. doi:10.1038/ nature 07205

16. Qi Q, Zhuang L, Shen Y, et al. A novel systemic inflammation response index (SIRI) for predicting the survival of patients with pancreatic cancer after chemotherapy. Cancer-Am Cancer Soc. 2016;122(14):2158-2167. doi:10.1002/cncr.30057

17. $\mathrm{Hu} \mathrm{M,} \mathrm{Xu} \mathrm{Q,} \mathrm{Yang} \mathrm{S,} \mathrm{et} \mathrm{al.} \mathrm{Pretreatment} \mathrm{systemic} \mathrm{inflammation}$ response index (SIRI) is an independent predictor of survival in unresectable stage III non-small cell lung cancer treated with chemoradiotherapy: a two-center retrospective study. Ann Transl Med. 2020;8(20):1310. doi:10.21037/atm-20-6484

18. Hong YM, Yoon KT, Hwang TH, Cho M. Pretreatment peripheral neutrophils, lymphocytes and monocytes predict long-term survival in hepatocellular carcinoma. Bmc Cancer. 2020;20(1):937. doi:10.1186/s12885-020-07105-8

19. Han D, Zhang J, Zhao J, et al. Platelet-to-lymphocyte ratio is an independent predictor of chemoradiotherapy-related esophageal fistula in esophageal cancer patients. Ann Transl Med. 2020;8(18):1163. doi:10.21037/atm-20-4053

20. Topkan E, Kucuk A, Ozdemir Y, et al. Systemic inflammation response index predicts survival outcomes in glioblastoma multiforme patients treated with standard stupp protocol. J Immunol Res. 2020;2020:8628540. doi:10.1155/2020/8628540

21. Feng Y, Zhang N, Wang S, et al. Systemic inflammation response index is a predictor of poor survival in locally advanced nasopharyngeal carcinoma: a propensity score matching study. Front Oncol. 2020;10:575417. doi:10.3389/fonc.2020.575417

22. Wang C, Wang M, Zhang X, et al. The neutrophil-to-lymphocyte ratio is a predictive factor for the survival of patients with hepatocellular carcinoma undergoing transarterial chemoembolization. Ann Transl Med. 2020;8(8):541. doi:10.21037/atm.2020.02.113

23. Chen Y, Jin M, Shao Y, Xu G. Prognostic value of the systemic inflammation response index in patients with adenocarcinoma of the oesophagogastric junction: a propensity score-matched analysis. Dis Markers. 2019;2019:4659048. doi:10.1155/2019/4659048

24. Chao B, Ju X, Zhang L, Xu X, Zhao Y. A novel prognostic marker systemic inflammation response index (SIRI) for operable cervical cancer patients. Front Oncol. 2020;10:766. doi:10.3389/ fonc. 2020.00766

25. Geng Y, Zhu D, Wu C, et al. A novel systemic inflammation response index (SIRI) for predicting postoperative survival of patients with esophageal squamous cell carcinoma. Int Immunopharmacol. 2018;65:503-510. doi:10.1016/j.intimp.2018.10.002
26. Koroki K, Ogasawara S, Ooka Y, et al. Analyses of intermediate-stage hepatocellular carcinoma patients receiving transarterial chemoembolization prior to designing clinical trials. Liver Cancer. 2020;9(5):596-612. doi:10.1159/000508809

27. Kudo M, Ikeda M, Ueshima K, et al. Response evaluation criteria in cancer of the liver version 5 (RECICL 2019 revised version). Hepatol Res. 2019;49(9):981-989. doi:10.1111/hepr.13394

28. Gaba RC, Lewandowski RJ, Hickey R, et al. Transcatheter therapy for hepatic malignancy: standardization of terminology and reporting criteria. J Vasc Interv Radiol. 2016;27(4):457-473. doi:10.1016/j.jvir.2015.12.752

29. Peisen F, Maurer M, Grosse U, et al. Predictive performance of the mHAP-II score in a real-life western cohort with hepatocellular carcinoma following trans-arterial chemoembolisation with drug-eluting beads (DEB-TACE). Eur Radiol. 2020;30 (7):3782-3792. doi:10.1007/s00330-020-06734-8

30. Habbel V, Zeile M, Stavrou GA, et al. Correlation between SACE (Subjective Angiographic Chemoembolization Endpoint) score and tumor response and its impact on survival after DEB-TACE in patients with hepatocellular carcinoma. Abdom Radiol. 2019;44 (10):3463-3479. doi:10.1007/s00261-019-02128-7

31. Pacheco-Barcia V, Mondejar SR, France T, et al. A systemic inflammation response index (SIRI) correlates with survival and predicts oncological outcome for mFOLFIRINOX therapy in metastatic pancreatic cancer. Pancreatology. 2020;20(2):254-264. doi:10.1016/j.pan.2019.12.010

32. Buonaguro L, Tagliamonte $\mathrm{M}$, Petrizzo A, Damiano E, Tornesello ML, Buonaguro FM. Cellular prognostic markers in hepatocellular carcinoma. Future Oncol. 2015;11(11):1591-1598. doi: $10.2217 /$ fon. 15.39

33. O'Rourke JM, Sagar VM, Shah T, Shetty S. Carcinogenesis on the background of liver fibrosis: implications for the management of hepatocellular cancer. World J Gastroenterol. 2018;24 (39):4436-4447. doi:10.3748/wjg.v24.i39.4436

34. Roth GS, Decaens T. Liver immunotolerance and hepatocellular carcinoma: patho-physiological mechanisms and therapeutic perspectives. Eur J Cancer. 2017;87:101-112. doi:10.1016/j.ejca.2017.10.010

35. Prieto J, Melero I, Sangro B. Immunological landscape and immunotherapy of hepatocellular carcinoma. Nat Rev Gastroenterol Hepatol. 2015;12(12):681-700. doi:10.1038/nrgastro.2015.173

36. Schobert IT, Savic LJ, Chapiro J, et al. Neutrophil-to-lymphocyte and platelet-to-lymphocyte ratios as predictors of tumor response in hepatocellular carcinoma after DEB-TACE. Eur Radiol. 2020;30 (10):5663-5673. doi:10.1007/s00330-020-06931-5

37. Mantovani A. The yin-yang of tumor-associated neutrophils. Cancer Cell. 2009;16(3):173-174. doi:10.1016/j.ccr.2009.08.014

38. Gao Q, Zhao YJ, Wang XY, et al. CXCR6 upregulation contributes to a proinflammatory tumor microenvironment that drives metastasis and poor patient outcomes in hepatocellular carcinoma. Cancer Res. 2012;72(14):3546-3556. doi:10.1158/0008-5472.CAN-11-4032

39. Bingle L, Brown NJ, Lewis CE. The role of tumour-associated macrophages in tumour progression: implications for new anticancer therapies. J Pathol. 2002;196(3):254-265. doi:10.1002/path.1027

40. Liu LZ, Zhang Z, Zheng BH, et al. CCL15 recruits suppressive monocytes to facilitate immune escape and disease progression in hepatocellular carcinoma. Hepatology. 2019;69(1):143-159. doi:10.1002/hep.30134

41. Mantovani A, Allavena P, Sica A. Tumour-associated macrophages as a prototypic type II polarised phagocyte population: role in tumour progression. Eur J Cancer. 2004;40(11):1660-1667. doi:10.1016/j. ejca.2004.03.016

42. Wieder T, Eigentler T, Brenner E, Rocken M. Immune checkpoint blockade therapy. J Allergy Clin Immunol. 2018;142(5):1403-1414. doi:10.1016/j.jaci.2018.02.042

43. Zhu Z, Xu L, Zhuang L, et al. Role of monocyte-to-lymphocyte ratio in predicting sorafenib response in patients with advanced hepatocellular carcinoma. Onco Targets Ther. 2018;11:6731-6740. doi:10.2147/OTT.S173275 


\section{Publish your work in this journal}

Risk Management and Healthcare Policy is an international, peerreviewed, open access journal focusing on all aspects of public health, policy, and preventative measures to promote good health and improve morbidity and mortality in the population. The journal welcomes submitted papers covering original research, basic science, clinical \& epidemiological studies, reviews and evaluations, guidelines, expert opinion and commentary, case reports and extended reports. The manuscript management system is completely online and includes a very quick and fair peer-review system, which is all easy to use. Visit http://www.dovepress.com/testimonials.php to read real quotes from published authors. 